\title{
Editorial: Cryptography and Communications, Volume 1, Issue 1
}

\author{
Claude Carlet
}

Received: 18 February 2009 / Accepted: 18 February 2009/Published online: 19 March 2009

(C) Springer Science + Business Media, LLC 2009

Boolean functions and sequences, and more generally all the discrete structures used in error correcting coding, cryptography or communications, are highly active areas of research, with an increasing number of international conferences devoted (or partly devoted) to them.

There exist journals covering the broader domain, but none that focus on these specific areas. And while there are conferences which address these specific research areas, the limited time for reviewing does not always allow referees to write detailed reviews, and substantial revisions improving the quality of the papers are rarely possible. In addition, page constraints often limit the potential quality. The lack of an appropriate vehicle backed by a rigorous selection process to disseminate research in these areas is a serious concern for the community.

In an effort to fill the existing gap, we have decided to found the journal "Cryptography and Communications - Discrete Structures, Boolean Functions and Sequences" (CCDS) to provide a forum for researchers where their work in cryptography, error correcting codes, communications and their interactions can converge. The core focus of the journal will be all of the discrete structures used in stream and block ciphers in symmetric cryptography and in error correcting codes; code division multiple access in communications; and random number generation for statistics, cryptography and numerical methods. In particular, research comprising Boolean functions and sequences, without excluding any other discrete structure used in cryptography and communications (e.g., finite fields and other algebraic structures), will be strongly encouraged.

The editorial board will work to ensure a combination of a high level of selection and a short review process, never compromising on the quality of the papers. We believe that achieving such seemingly contradictory goals is possible when the scope of the journal remains intently focussed.

I wish to express my gratitude to Harald Niederreiter, who participated throughout the discussions on the founding of this journal. I am also indebted to Subhamoy Maitra who

C. Carlet ( $\square)$

Department of Mathematics, Université Paris 8, 2, rue de la Liberté, 93526 Saint Denis Cedex 02,

France

e-mail: claude.carlet@inria.fr 
had the original idea of the journal and to Cunsheng Ding, Guang Gong, Tor Helleseth and Andrew Klapper who participated in the original advisory board. Many thanks to them and to all the members of the editorial board.

I deeply thank Springer for welcoming our journal; many thanks to Jennifer Evans and Melissa Fearon for their great help.

Finally, I wish to thank Charles Colbourn, Joan Daemen, Thomas Johansson, Harald Niederreiter and Vincent Rijmen for having accepted our proposal to submit their papers to the opening issue of this journal. 